gender, and absence of fibromyalgia. This temporary approach has been a feasible compensation for face-to-face visits, thus reassuring for future months before the end of pandemic.

Disclosure of Interests: Francesca Ingegnoli: None declared, Gilberto Cincinelli: None declared, Angela Flavia Luppino: None declared, Ennio Favalli Speakers bureau: AbbVie, Sanofi-Genzyme, Lilly, UCB, Pfizer, Novartis, Janssen, Paid instructor for: Roche, MSD, Consultant of: Lilly, Galapagos, Roberto Caporali Speakers bureau: Abbvie, Amgen, BMS, Celltrion, Galapagos, Gilead, Lilly, Pfizer, Roche, UCB, Sanofi, Fresenius Kabi, Samsung bioepis, MSD, Consultant of: Galapagos, Gilead, Lilly,Janssen, MSD.

DOI: 10.1136/annrheumdis-2021-eular.1104

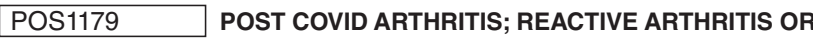 RHEUMATIC DISEASE FLARE OR BOTH}

F. Fayed ${ }^{1}$, E. Abdelkarim ${ }^{1} .{ }^{1}$ Alexandria University Students' Hospital,

Rheumatology and immunology department, alexandria, Egypt

Background: Reactive arthritis $(\mathrm{Re} A)$ is an emerging arthritis after viral infection especially respiratory and gastrointestinal related viruses. In 2020, SARS-COV2 virus is sweeping worldwide with diverse symptoms and prolonged post-Covid manifestations in which are arthralgia and myalgia are frequently present for days and even months. Interestedly, arthritis in covid era is a part of ReA or a flare of autoimmune disease, it is challenging. ${ }^{(1)}$

Objectives: Our objective was to determine the frequency of musculoskeletal symptoms with SARS-COV2 virus and after recovery as well as its relation to autoimmune diseases flares.

Methods: A Prospective study was done on 241 patients who admitted to the Rheumatology clinic from March 2020 to January 2021, complaining from new onset of musculoskeletal symptoms. Detailed history, Examination of systems including Musculoskeletal, laboratory investigation, past history of existing rheumatic disease, and history of infection with covid 19.

Results: Among 241 patients with median age 34.4, 36.92\% had an existing Rheumatic disease while $63.08 \%$ are not. Moreover, $39 \%$ of patients had a post history with covid 19 within weeks. The most frequent Musculoskeletal symptoms are myalgia (74.56\%), arthralgia (69.36\%), and arthritis (10.78\%). Furthermore, ReA (Post covid arthritis) accounted (2.07\%) especially monoarthritis of Ankle $(68.75 \%)$ while rheumatic diseases flares $(24.48 \%)$ as well as new onset rheumatic diseases $(39.68 \%)$. ( $p \leq 0.001$ ) patients of ReA improved on NSAID and intrarticular injection of glucocorticosteroid.

Conclusion: Covid 19 is one of environmental triggers for development of rheumatic disease as well as reactive arthritis post viral infections. ReA is commonly affected the Ankle joint mainly monoarthritis and had improved on oral NSAID and intrarticular injection of Glucocorticosteroid.

REFERENCES:

[1] Schett, G., Manger, B., Simon, D. et al. COVID-19 revisiting inflammatory pathways of arthritis. Nat Rev Rheumatol 16, 465-470 (2020).

Disclosure of Interests: None declared.

DOI: 10.1136/annrheumdis-2021-eular. 1148

\section{POS1180 RHEUMATOID ARTHRITIS DURING THE FIRST WAVE OF THE SARS-CoV-2/COVID-19 PANDEMIC IN PORTUGAL: RESULTS FROM THE COVID IN RA (COVIDRA) SURVEY}

F. Araujo ${ }^{1}$, N. Gonçalves ${ }^{2}$, A. F. Mourão $0^{1,2} .{ }^{1}$ Hospital de Sant'Ana, Rheumatology and Osteoporosis Unit, Parede, Portugal; ${ }^{2}$ Hospital Egas Moniz, Department of Rheumatology, Lisboa, Portugal

Background: The outcomes of the infection by the SARS-CoV-2 in patients with immune-mediated inflammatory diseases were largely unknown during the early days of the COVID-19 pandemic. It was hypothesized that these patients were at higher risk of morbidity and mortality due to their inherent immune dysfunction and immunosuppressive therapy. Several rheumatology societies issued recommendations urging patients not to stop their anti-rheumatic treatments.

Objectives: To assess treatment compliance of patients with rheumatoid arthritis (RA) during the first wave of the SARS-CoV-2/COVID-19 pandemic in Portugal. Methods: The web-based survey COVIDRA (COVID in RA) was developed to assess the impact of the first wave mandatory confinement in patients with RA focusing on 5 domains: RA symptoms, attitudes towards medication, employment status, physical exercise and mental health. The questionnaire was sent to RA patients through e-mail and social media of the Portuguese Society of Rheumatology and two patient associations; and it was filled locally at two rheumatology centers in Lisbon. Recruitment took place during June and July 2020. Descriptive statistics were generated by the survey software and were afterwards transported and evaluated using appropriate biostatistics software.
Results: We obtained 441 valid questionnaires. Most respondents were female $(88.4 \%)$, caucasian $(93.6 \%)$, with a mean age of $58(+/-13)$ years. The majority $(57.6 \%)$ had longstanding disease ( $>10$ years) and were treated with csDMARDs $(63.2 \%)$ and/or bDMARDs/tsDMARDS (23,7\%). Only 14\% ( $\mathrm{N}=61)$ discontinued or reduced the dosage or frequency of their RA treatment. Most of these changes were previously planned by the attending physician (27.9\%). Only 11 patients (18\%) discontinued their immunosuppressive medication out of fear of becoming infected with SARS-CoV-2 (corresponding to $2.5 \%$ of total responders). Another 11 patients did so because they had no prescription, couldn't go to the community/hospital pharmacy or couldn't afford the medication. Although these numbers preclude any statistical analysis, when compared to patients who persisted on their treatment, those discontinuing due to fear of contagion were younger (56.4 vs 58.5 years), all female (100 vs $86.8 \%$ ), with long-lasting disease ( $\geq 11$ years) $(90.9 \%$ vs $57.5 \%)$, more frequently treated with bDMARDs (36.4 vs $23.1 \%$ ) and presenting more symptoms of depression ( 54.5 vs $49.7 \%$ ).

Conclusion: Most RA patients complied with their treatment during the first wave of the SARS-CoV-2 pandemic in Portugal. Only a minority changed their immunosuppressive treatment due to fear of SARS-CoV-2 infection. Very similar rates of immunosuppressive discontinuation due to fear of contagion were reported by other authors (such as Schmeiser et al, Pineda-sic et al and Fragoulis et al). Disclosure of Interests: Filipe Araujo Speakers bureau: Pfizer, Biogen, Novartis, Menarini, Consultant of: MSD, Nuno Gonçalves: None declared, Ana Filipa Mourão: None declared.

DOI: 10.1136/annrheumdis-2021-eular.1152

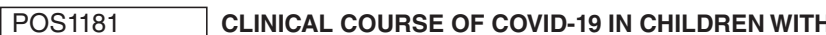 RHEUMATIC DISEASE UNDER BIOLOGIC THERAPY}

F. Demir ${ }^{1}$, K. Ulu ${ }^{1}$, Ş. Çağlayan ${ }^{1}$, T. Coşkuner ${ }^{1}$, B. Sözeri ${ }^{1} .{ }^{1}$ Health Sciences University, Umraniye Training and Research Hospital, Pediatric Rheumatology, Istanbul, Turkey

Background: Since the beginning of the COVID-19 pandemic in our country, Turkey, more than two million people have been infected and more than 20,000 people have died. Although children infected less frequently and generally have a milder findings of COVID-19, the number of patients with a more severe clinical course as multisystem inflammatory syndrome in children (MIS-C) is increasing significantly. However, it has not been shown exactly how biological disease-modifying antirheumatic drug (bDMARD)s, which we frequently use in our pediatric rheumatology practice, and/or the underlying rheumatological diseases affect the clinical course of COVID-19.

Objectives: Here, we aimed to reveal the outcome of COVID-19 infection in our patients with pediatric rheumatic disease and treated with bDMARDs.

Methods: During the period between April 1, 2020 and December 1, 2020, the patients who received bDMARDs were evaluated at the regular outpatient clinic follow-up or by telemedicine with a maximum of 3 months interval. Clinical and demographic characteristics, COVID-19 data and outcome of these patients were retrospectively collected.

Results: Out of the 436 patients treated with bDMARDs, 39 children were infected with COVID-19. The diagnosis was confirmed in 37 patients by RT-PCR (nasalpharyngeal swab) and in two by antibody test. Twenty-two $(56.4 \%)$ patients were female $(17$ male, $\% 43.6)$ and the median age of patients were 12.3 years (min-max: $1.2-20.9$ ). The primary diagnosis of patients were as follows; 20 juvenile idiopathic arthritis (six were systemic subtype), 12 systemic autoinflammatory diseases, three vasculitis, three chronic recurrent multifocal osteomyelitis and one Sjögren's syndrome. Prior to COVID-19 infection, 13 patients $(33.3 \%)$ were using canakinumab, seven were infliximab $(18 \%)$ five were adalimumab (12.8\%), four were etanercept $10.2 \%$ ), four were tocilizumab $(10.2 \%)$, three were anakinra $(7.7 \%)$, two were rituximab $(5.1 \%)$, and one was tofacitinib (2.6\%)

Of the 39 patients, 21 had at least one COVID-19-related symptom, while 18 patients were asymptomatic. No laboratory or imaging tests was performed for asymptomatic patients and they were followed up without treatment at home isolation. Laboratory tests revealed that fourteen patients had elevated acute phase reactants, six had elevated D-dimer levels, three had lymphopenia $\left(<1000 / \mathrm{mm}^{3}\right)$ and three had hyperferritinemia.

Hospitalization was required in 20 patients $(51.3 \%)$ at median of 7 -days (minmax: $3-17$ ) and pediatric intensive care unit admission in one. Five patients developed MIS-C and one of these patients was followed up in the pediatric intensive care unit. Myocardial dysfunction was developed in this patient and he was died. The other four patients fully recovered with no remain morbidity.

Conclusion: Considering the literature data and the results of our study, it is not possible to say that currently used bDMARDs worse the course of COVID19 infection. In patients with underlying risk factors for hyperinflammation, as in one of our patients, COVID-19 may cause mortality regardless of the use of bDMARDs. Whether bDMARDs does not affect the severity of the disease, but it is still not true to say that these drugs are protective. Since cessation of 